\title{
CORRECTION
}

\section{Correction to: Osteoprotegerin/bone morphogenetic protein 2 combining with collagen sponges on tendon-bone healing in rabbits}

\author{
Bing Wei ${ }^{1} \cdot$ Chao Wang ${ }^{1} \cdot$ Cheng Yan ${ }^{1} \cdot$ Bushun Tang ${ }^{1} \cdot$ Xiaofei Yu $^{2} \cdot$ Hui Zhang ${ }^{3} \cdot$ Lixia Tang $^{3}$ (D) $\cdot$ Qing Wang $^{1}$ (I)
}

Published online: 17 December 2021

(c) The Japanese Society Bone and Mineral Research 2021

\section{Correction to: \\ Journal of Bone and Mineral Metabolism (2020) 38:432-441 \\ https://doi.org/10.1007/s00774-019-01078-w}

In the original publication of the article, Fig. 4A and 4B are duplicate. The correct Fig. 4A is provided in this correction.

The original article can be found online at https://doi.org/10.1007/ s00774-019-01078-w.

Lixia Tang

tanglixia138@163.com

$\triangle$ Qing Wang

wangqinglijun@163.com

1 Department of Orthopedics, The First People's Hospital of Yongkang, Jinhua 321300, People's Republic of China

2 Department of Pathology, The First People's Hospital of Yongkang, Jinhua 321300, People's Republic of China

3 Department of General Diseases, The First People's Hospital of Yongkang, Jinhua 321300, People's Republic of China 


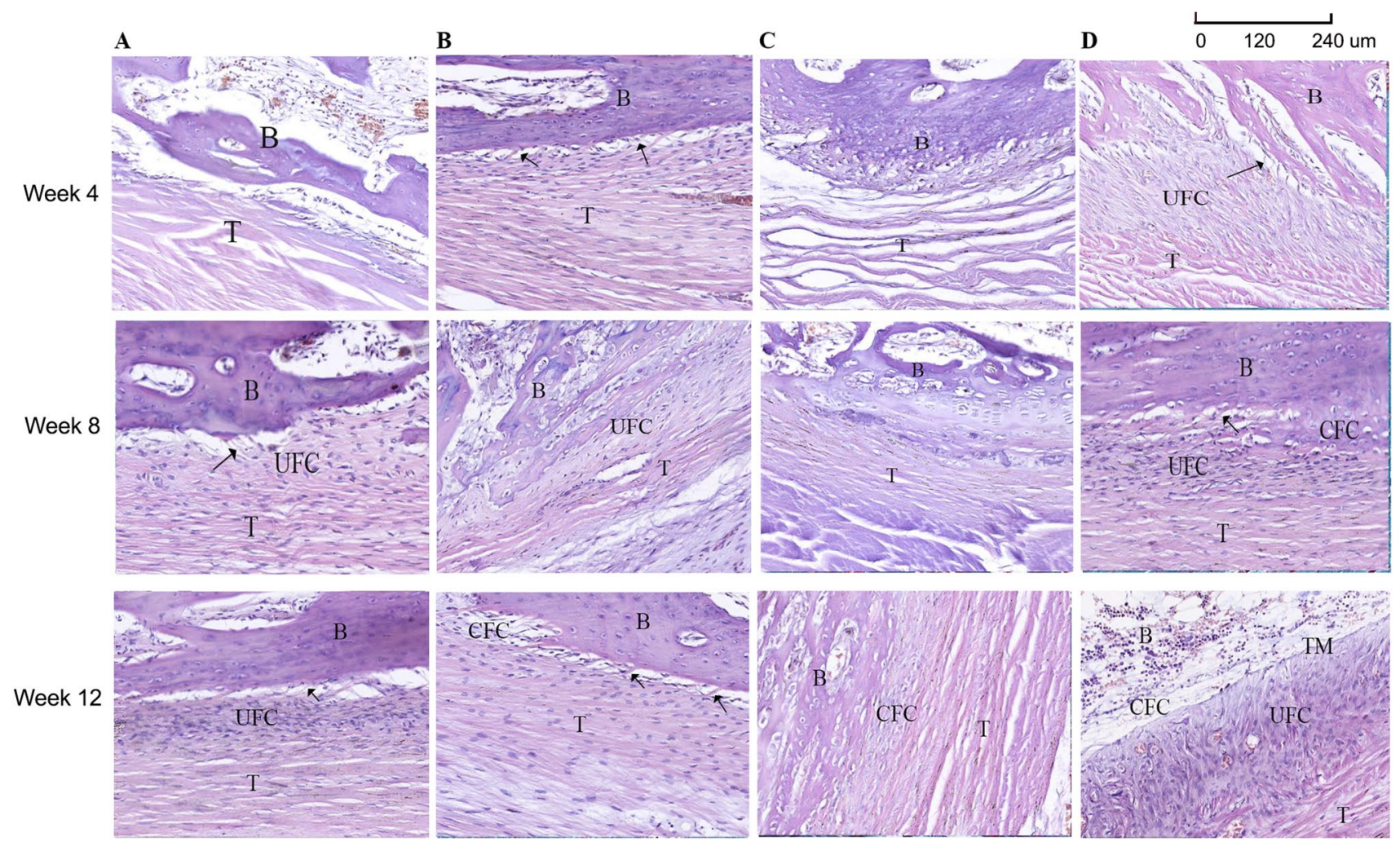

In addition, the term "at 12 weeks" were incorrectly included in the captions of Fig. 4 and 5.

The correct captions should read as below.

Caption of Fig. 4: Tissue morphology post operation (Hematoxylin and Eosin, $\times 100$ ). $T$ tendon, $B$ bone, $U F C$ uncalcified fibrocartilage, $C F C$ calcified fibrocartilage, $T M$ Tide mark; A black arrow: Sharpey-like collagen fibers. a the blank control group; $\mathbf{b}$ the OPG/BMP-2; $\mathbf{c}$ the CS group; d the OPG/BMP-2/CS group.

Caption of Fig. 5: M-T staining $(\times 100)$ shows fibrocartilage and collagen fiber formation around the tendon. $T$ tendon, $B$ bone, $F C$ fibrocartilage, $C F$ collagen fibers. a the blank control group; $\mathbf{b}$ the OPG/BMP-2; $\mathbf{c}$ the CS group; $\mathbf{d}$ the OPG/BMP-2/CS group.

Publisher's Note Springer Nature remains neutral with regard to jurisdictional claims in published maps and institutional affiliations. 